\title{
Design and in vivo evaluation of emulgel formulations including green tea extract and rose oil
}

\author{
EVREN ALGIN YAPAR ${ }^{1 *}$ \\ ÖZGE INAL ${ }^{2}$ \\ M. SEDEF ERDAL ${ }^{3}$ \\ ${ }^{1}$ The Ministry of Health of Turkey \\ Turkish Medicines and Medical Devices \\ Agency, Sögütözü Mahallesi, 2176, 06520 \\ Çankaya-Ankara, Turkey \\ ${ }^{2}$ Department of Pharmaceutical \\ Technology, Faculty of Pharmacy \\ University of Ankara, 06100 Tandoğan- \\ -Ankara, Turkey \\ ${ }^{3}$ Department of Pharmaceutical Technology \\ Faculty of Pharmacy, Istanbul University \\ 34116 Beyazlt-Istanbul, Turkey
}

Accepted September 25, 2013
Prevention of skin aging and its treatment is an emerging field for development of new formulations in cosmetics. Accordingly, plant extracts with antioxidant properties are beneficial cosmetic ingredients for this purpose. This study was aimed at developing a stable and easily manufactured emulgel including green tea extract and rose oil that is effective on the barrier function and hydration of the skin. An emulgel formulation containing $20 \%$ green tea extract and $5 \%$ rose oil was designed as a result of pre-formulation studies. Physicochemical characterization, in vitro stability studies, in vivo water content of the stratum corneum and transepidermal water loss studies were carried out afterwards. In vivo studies on ten female subjects were evaluated by using non-invasive skin bioengineering techniques. Finally, a cosmetically acceptable, stable and effective emulgel formulation for skin barrier function with good hydrating properties was obtained for skin hydration, protection and anti-aging purposes.

Keywords: green tea extract, rose oil, emulgel, skin water content, transepidermal water loss, biophysical measurements

Plant materials with antioxidant and skin anti-aging properties have been widely used in cosmetic products for many years. Among them Camellia sinensis (L.) (green tea) extract is known for its biological and pharmacological activities attributed mainly to green tea catechins, which are polyphenolic compounds present in unfermented dried leaves of $C$. sinensis $(1,2)$. A short heating procedure of green tea leaves led to inactivation of polyphenol oxidase, thereby preserving the antioxidant activities of polyphenols. The total content of polyphenols in tea leaves varies from approximately 20 to $40 \%$, depending on the subspecies of the plant and geographic location (3). The four major polyphenolic catechins present in green tea leaves are (2)-epicatechin (EC), epigallocatechin

* Correspondence; e-mail: evren.yapar@yahoo.com 
(EGC), (2)-epicatechin-3-gallate and epigallocatechin-3-gallate (EGCG). EGCG is the most abundant and active polyphenol in green tea $(3,4)$.

An early study had already proved certain effects of a cream containing green tea extract on the viscoelastic properties of the skin (5). In another study, a 60-day treatment of green tea extract showed anti-wrinkle efficacy in healthy subjects and significant improvements have been observed on skin roughness, scaliness and wrinkling (2). Studies on an in vitro model of human skin fibroblasts showed the protective effect of green tea extract from toxic reactive oxygen species (6). In addition to its anti-oxidative and anti-ageing potentials, green tea may exert other therapeutic activities such as anti-cancer, anti-inflammatory, anti-microbial, and anti-acne activities $(1,3,7)$.

Topical in vivo applications of rose oil from the petals of Rosa damascena (Miller) have been reported to improve skin barrier function and promote keratinocyte differentiation (8). Furthermore, antioxidant and antimicrobial activities of rose oil were observed (9-11).

Antioxidants are introduced as primary ingredients especially into daytime cosmetics to scavenge free radicals produced by ultraviolet light (UV) and environmental pollutants, which are involved in skin ageing processes (11). Green tea is apparently capable of providing skin protection against sun damage by quenching the skin with free radicals (12-14). Human skin penetration and retention of EGCG from green tea extracts has been found sufficient to exert positive effects on the skin (15).

In case of pharmaceutical forms, emulgels are emulsions that are gelled by mixing with a gelling agent; they possess advantages of both emulsions and gels (16). Briefly, ease of spreadability, convenience of viscosity and appearance and emollient effect can be summarized as advantages of emulgels.

The objective of the present work is to design an emulgel formulation that includes green tea leave extracts and rose essential oil because of the above mentioned effects of these natural ingredients. For this purpose, emulgel formulations in pre-formulation studies were evaluated in terms of $\mathrm{pH}$ and viscosity. Organoleptic controls and microscopic analyses were performed before and after a stability period of 6 months. According to the results, the most stable formulation (F9A) containing $5 \%$ rose oil and $20 \%$ green tea extract was chosen for in vivo experiments. Biophysical measurements were used in order to evaluate the effect of the emulgel on skin hydration and transepidermal water loss (TEWL) after a single and a 5-day-long period of multiple applications.

\section{EXPERIMENTAL}

\section{Materials}

Carbopol 1342 and Carbopol 934 were provided by BF Goodrich, Belgium. Glyceryl monostearate, methylparaben, and propylparaben were purchased from Sigma-Aldrich, USA. Cetostearyl alcohol, liquid paraffin, glycerine and triethanolamine were obtained from Merck, Germany. Dimethicone was provided from Johnson and Johnson, Turkey. Trademark products of rose oil (Shiffa Home, Turkey) and green tea leaves (Çaykur, Turkey) were purchased from a local market (Ankara, Turkey). 
E. Algin Yapar et al.: Design and in vivo evaluation of emulgel formulations including green tea extract and rose oil, Acta Pharm. 63 (2013) 531-543.

\section{Preparation of formulations}

Emulgels were prepared in two steps, i.e., step I and step II. In step I, the gel phase was prepared; in step II, the gel phase was combined with the oily phase in order to give the final emulgel formulation. Step I and step II procedures were as follows:

\section{Gel phase preparation}

Briefly, the gel base was prepared by dispersing Carbopol 934 or Carbopol 1342 in $30 \mathrm{~mL}$ water using a magnetic stirrer in order to obtain a homogeneous gel base. Triethanolamine solution $(5 \%, \mathrm{~m} / \mathrm{m})$ was used to neutralize the $\mathrm{pH}$ and air bubbles were removed using an ultrasonic water bath (Ultrasonic LC 30, Germany) for $1 \mathrm{~h}$. The Carbopol 934 gel phase was featured in formulations F1-F4 whereas Carbopol 1342 was featured in F5-F10. The codes and ingredients of formulations F1-F10 are given in Table I.

Table I. Content of the formulations $(\%, \mathrm{~m} / \mathrm{m})$

\begin{tabular}{|c|c|c|c|c|c|c|c|c|c|c|c|}
\hline & Ingredient & $\mathrm{F} 1$ & F2 & F3 & $\mathrm{F} 4$ & $\mathrm{~F}^{\mathrm{a}}$ & F6 & F7 & F8 & $\begin{array}{c}\text { F9A/ } \\
\text { F9B }\end{array}$ & $\begin{array}{c}\text { F10A/ } \\
\text { F10B }\end{array}$ \\
\hline \multirow{6}{*}{ 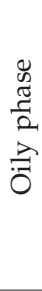 } & $\begin{array}{l}\text { Glycerine } \\
\text { monostearate }\end{array}$ & 1 & 1 & 1 & 1 & 1 & 1 & 1 & 1 & 1 & 1.5 \\
\hline & Cetostearyl alcohol & 3.5 & 3.5 & 3.5 & 3.5 & 3.5 & 3.5 & 3.5 & 3.5 & 3.5 & 4 \\
\hline & Liquid paraffin & 12 & 12 & 12 & 12 & 12 & 8 & 12 & 8 & 12 & 8 \\
\hline & Dimeticone & 0.3 & 0.3 & 0.3 & 0.3 & 0.3 & 0.3 & 0.3 & 0.3 & 0.3 & 4 \\
\hline & Rose oil & - & - & - & - & - & 5 & - & 5 & 5 & 5 \\
\hline & Propyl paraben & 0.25 & 0.25 & 0.25 & 0.25 & 0.25 & 0.25 & 0.25 & 0.25 & 0.25 & 0.25 \\
\hline \multirow{7}{*}{ 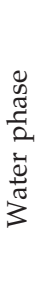 } & Methyl paraben & 0.25 & 0.25 & 0.25 & 0.25 & 0.25 & 0.25 & 0.25 & 0.25 & 0.25 & 0.25 \\
\hline & Glycerine & 3 & 3 & 3 & 3 & 3 & 3 & 3 & 3 & 3 & 3 \\
\hline & Carbopol 934 & 0.3 & 0.2 & 0.2 & 0.2 & - & - & - & - & - & - \\
\hline & Carbopol 1342 & - & - & - & - & 0.3 & 0.3 & 0.3 & 0.3 & 0.3 & 0.3 \\
\hline & Triethanolamine & 0.18 & 0.18 & - & - & 0.3 & 0.3 & 0.3 & 0.3 & 0.3 & 0.3 \\
\hline & Green tea extract $(\mathrm{mL})$ & - & - & - & 20 & - & - & 20 & 20 & 20 & 20 \\
\hline & Purified water (q.s.) & 100 & 100 & 100 & 100 & 100 & 100 & 100 & 100 & 100 & 100 \\
\hline
\end{tabular}

a F5 was used as Base Cream in studies in vivo.

\section{Emulgel preparation}

Oil and water phases (Table I) were heated separately to $65^{\circ} \mathrm{C}$ using a thermostatic water bath (Heraeus, Germany) and then mixed by stirring on a magnetic stirrer (250 rpm) (Osaka, Japan). After an emulsion was formed, the gel phase was added under continuous stirring. At that stage, an ultrasonic homogenizer (70 W, Bandelin Sanopuls HD 2070, Germany) was used for formulations F9B and F10B as a second preparation method. 
E. Algin Yapar et al.: Design and in vivo evaluation of emulgel formulations including green tea extract and rose oil, Acta Pharm. 63 (2013) 531-543.

In formulations F4 and F7-F10, green tea extract (20 g) produced from green tea leaves $(20 \%, \mathrm{~m} / \mathrm{m})$ by a simple decoction method were added to the water phase of the emulsion (17). In formulations F6 and F8-F10, $5 \%$ rose oil was added to the final emulgel when its temperature decreased to $30^{\circ} \mathrm{C}$.

\section{Characterization of emulgels}

Emulgels were characterized by performing organoleptic controls, $\mathrm{pH}$, viscosity and microscopic analysis after the preparation and during the stability period.

\section{Organoleptic controls}

Organoleptic controls involving oily feel, spreadability, stickiness and ease of absorption were evaluated as 0 (unavailable), + (moderate),++ (good),+++ (best). In addition, water dilution characteristics, homogeneity, odor and color of the formulations were evaluated.

\section{pH measurements}

The $\mathrm{pH}$ measurements were done using a pH-meter (Inolab WTW Series $\mathrm{pH} 720$, Germany) at room temperature $\left(24 \pm 1{ }^{\circ} \mathrm{C}\right)$ after 24 hours and six months after preparation. Samples were prepared by dilution of 1 gram of emulgel to $10 \mathrm{~mL}$ with purified water before the experiment. Measurements were taken in triplicates.

\section{Viscosity measurements}

Viscosity measurements and flow characteristics of the formulations were evaluated using a rotational viscometer (Brookfield DVII, TF spindle, $25 \pm 1{ }^{\circ} \mathrm{C}$ ). Measurements were taken in triplicates.

\section{Microscopic analyses}

Microscopic analyses were done using an optical microscope with 10x magnification (Leica DM 400, Germany). Samples were carefully diluted 1:1 with purified water, if dilution was required.

\section{Stability tests}

Formulations F1-F8 were stored in a cabin (Nüve ID 501, Turkey) of $25 \pm 1{ }^{\circ} \mathrm{C}$ temperature and $50 \pm 5 \%$ relative humidity for 6 months. Viscosity and microscopic changes were examined periodically.

Accelerated stability tests were performed to determine the stability of formulations F9A, F9B, F10A and F10B under accelerated gravitational conditions as stated below $(18-20)$ :

Freeze-thaw (F/T) cycling. - In the first cycle, formulations were kept at $+4{ }^{\circ} \mathrm{C}$ in 25 $\mathrm{mL}$ glass bottles for $24 \mathrm{~h}$ and then thawed at $25^{\circ} \mathrm{C}$ for $24 \mathrm{~h}$. In the second cycle, samples 
E. Algin Yapar et al.: Design and in vivo evaluation of emulgel formulations including green tea extract and rose oil, Acta Pharm. 63 (2013) 531-543.

kept at $+4{ }^{\circ} \mathrm{C}$ for another $24 \mathrm{~h}$ were thawed at $40{ }^{\circ} \mathrm{C}$ for $1.5 \mathrm{~h}$. Afterwards, samples were equilibrated under room conditions for $1 \mathrm{~h}$ before viscosity measurements and microscopic analyses were done. Control measurements were performed on samples kept at $25{ }^{\circ} \mathrm{C}$. All formulations featured in freeze-thaw cycling stability were evaluated after $5 \mathrm{~h}$ of centrifugation of $1500 \mathrm{~g}$ in terms of viscosity and microscopically.

Centrifugation method. - Centrifugation test was performed at $1500 \mathrm{~g}(\sim 3750 \mathrm{rpm})$ in a $10 \mathrm{~mL}$ tube using a laboratory centrifuge (Hettich Rotofix II, UK) at room temperature $\left(25 \pm 1^{\circ} \mathrm{C}\right)$. Inspection was done for possible phase separation after 15, 30, 60 minutes and 5 hours (19).

\section{In vivo studies}

The in vivo studies were carried out in an examination room under constant environmental conditions of $40 \pm 5 \%$ relative humidity and $21 \pm 1{ }^{\circ} \mathrm{C}$. Ten female volunteers, of 24 to 39 years of age, were selected and signed the Terms of Informed Consent after being informed about the objectives and research methods. Measurements were performed in accordance with the guidelines of the Standardization Group of the European Society of Contact Dermatitis (21).

Single application. - Emulgel formulation F9A and the Base Cream (formulation F5 was chosen as base; Table I) were applied non-occlusively to the volar side of the forearm of volunteers (10 female volunteers aged from 24-39 years). Skin water content and TEWL were determined with a Corneometer and Tewameter (Courage \& Khazaka electronic $\mathrm{GmbH}$, Cologne, Germany), respectively. A single dose corresponding to $500.0 \mathrm{mg}$ of each formulation was applied to the defined test area partitioned into two test fields using a self-adhesive tape $(30 \times 3 \mathrm{~cm})$. After 15 minutes, the formulation that was still on the skin was gently wiped off. The stratum corneum water content and skin barrier function were evaluated by biophysical measurements before application (baseline values) and 15, 30, 60, 120, 180 and 240 minutes after removal of the formulation.

Multiple applications. - Five volunteers (24-39 years old) were asked to apply the emulgel formulation F9A and the Base Cream twice daily over 5 days. At the end of the treatment period, their skin water content and transepidermal water loss (TEWL) were measured with the skin capacitance meter Corneometer CM 825 (Courage \& Khazaka) and Tewameter TM 210 (Courage\&Khazaka), respectively.

The stratum corneum moisture content was determined with a non-invasive technique using the Corneometer. Five individual measurements were carried out for each formulation at any time point and the mean of the measured hydration values was calculated. Changes in skin hydration were detected as Corneometer units. The rate of TEWL, as an indicator of skin barrier integrity, was determined using the Tewameter with a measuring time of $1 \mathrm{~min}$ for each formulation at any time point. The value with the lowest standard deviation was taken for comparison of the formulations. Before measurements were taken, the participants were acclimatized for at least 30 minutes in the examination room under constant environmental conditions mentioned above. Volunteers were requested not to use moisturizers, body lotions, soaps or occlusive cosmetic preparations on the tested skin areas $12 \mathrm{~h}$ prior to the in vivo study. 


\section{RESULTS AND DISCUSSION}

Results of the organoleptic evaluation of emulgel formulations F1-F10 are presented in Table II. Green tea extract gave a slightly green color to the formulations. F9B and $\mathrm{F} 10 \mathrm{~B}$, formulations produced by the sonication process, had opaque-greenly color in their final form. Emulgel F5 showed the best spreadability and homogeneity among the first five formulations and therefore this formulation was chosen as the vehicle (Base Cream) for green tea extract and rose oil for further experiments. Emulgel formulations F6-F10 derived from F5 showed good spreadability and homogeneity characteristics after being loaded with green tea extract and/or rose oil.

Table II. Organoleptic controls at the end of 24 hours $^{a}$

\begin{tabular}{lcccccccccccc}
\hline Control & F1 & F2 & F3 & F4 & F5 & F6 & F7 & F8 & F9A & F9B & F10A & F10B \\
\hline Oily feel $^{\text {b }}$ & 0 & 0 & 0 & 0 & 0 & 0 & 0 & 0 & 0 & 0 & 0 & 0 \\
Spreadability & ++ & ++ & ++ & ++ & +++ & +++ & +++ & +++ & +++ & +++ & +++ & +++ \\
Ease of absorption & ++ & ++ & ++ & ++ & ++ & ++ & ++ & ++ & ++ & ++ & ++ & ++ \\
Water dilution & ++ & ++ & ++ & ++ & ++ & ++ & ++ & ++ & ++ & ++ & ++ & ++ \\
Stickiness & + & + & + & + & ++ & ++ & ++ & ++ & ++ & ++ & ++ & ++ \\
Homogeneity & 0 & + & + & 0 & ++ & ++ & ++ & +++ & +++ & +++ & +++ & +++ \\
Odor & + & + & + & + & + & + & + & + & + & + & + & + \\
Color & $\mathrm{C}$ & $\mathrm{W}$ & $\mathrm{W}$ & $\mathrm{SG}$ & $\mathrm{W}$ & $\mathrm{W}$ & $\mathrm{SG}$ & $\mathrm{SG}$ & $\mathrm{SG}$ & $\mathrm{OSG}$ & $\mathrm{SG}$ & OSG \\
\hline
\end{tabular}

a 0 (unavailable), + (moderate), ++ (good), +++ (best)

b All the formulations gave an oily feel the first moment after application but after a few seconds this feeling changed to a non-oily feel.

c C - Cream, W - White, SG - Slightly green, OSG - Opac and slightly green

According to the results of $\mathrm{pH}$ analysis presented in Table III, when the gel was not neutralized during preparation (F3 and F4), the finished emulgel product had also a lower $\mathrm{pH}$ value $(5.91 \pm 0.025$ and $6.62 \pm 0.055$, respectively) compared to that of emulgel formulations F1 and F2 based on Carbopol 934.

Emulgel formulations F5-F10 were prepared with a neutralized gel phase based on Carbopol 1342. After the addition of the oil phase, the $\mathrm{pH}$ of emulgel formulations was in the range of $5.91 \pm 0.025$ and $7.35 \pm 0.030$. The $\mathrm{pH}$ value declined at the end of 6 months with some variations and ranged from $5.30 \pm 0.03$ to $6.75 \pm 0.09$, which is considered acceptable for avoiding the risk of skin irritation. The oxidation process and the formation of oxidation products in the oil phase might be the reason for the slight reduction in $\mathrm{pH}$ values.

Neutralizing the gel phase before adding the emulsion phase was not necessary only for appropriate $\mathrm{pH}$, but also for achieving maximum viscosity in the final emulgel formulation. In our study, emulgel F5 showed the highest viscosity among the first five formulations. At the end of 6 months of storage, the decrease in viscosity of formulations F6 and F8 was probably associated with the presence of rose oil in the formulation (Fig. 1a). Increase in viscosity followed the order F4 $>$ F1 $>$ F5 $>$ F3 $>$ F2 = F7. As shown 
E. Algin Yapar et al.: Design and in vivo evaluation of emulgel formulations including green tea extract and rose oil, Acta Pharm. 63 (2013) 531-543.

Table III. $p H$ of the prepared formulations at different time intervals

\begin{tabular}{cccc}
\hline Formulation & Gel phase $(24 \mathrm{~h})$ & Emulgel $(24 \mathrm{~h})$ & Emulgel $(6$ months $)$ \\
\hline F1 & $6.60 \pm 0.050$ & $7.18 \pm 0.035$ & $6.25 \pm 0.030$ \\
F2 & $5.95 \pm 0.060$ & $6.75 \pm 0.040$ & $6.30 \pm 0.025$ \\
F3 & $3.50 \pm 0.031$ & $5.91 \pm 0.025$ & $5.30 \pm 0.025$ \\
F4 & $3.50 \pm 0.045$ & $6.62 \pm 0.055$ & $5.83 \pm 0.060$ \\
F5 & $6.20 \pm 0.068$ & $7.18 \pm 0.075$ & $6.75 \pm 0.085$ \\
F6 & $6.20 \pm 0.025$ & $7.35 \pm 0.030$ & $6.54 \pm 0.025$ \\
F7 & $6.20 \pm 0.030$ & $7.24 \pm 0.060$ & $6.29 \pm 0.015$ \\
F8 & $6.03 \pm 0.082$ & $6.87 \pm 0.045$ & $5.86 \pm 0.065$ \\
F9A & $6.06 \pm 0.029$ & $6.91 \pm 0.025$ & $5.98 \pm 0.030$ \\
F9B & $6.04 \pm 0.040$ & $6.77 \pm 0.070$ & $6.04 \pm 0.075$ \\
F10A & $6.04 \pm 0.061$ & $7.06 \pm 0.032$ & $6.14 \pm 0.035$ \\
F10B & $6.01 \pm 0.035$ & $7.11 \pm 0.065$ & $6.30 \pm 0.050$ \\
\hline
\end{tabular}
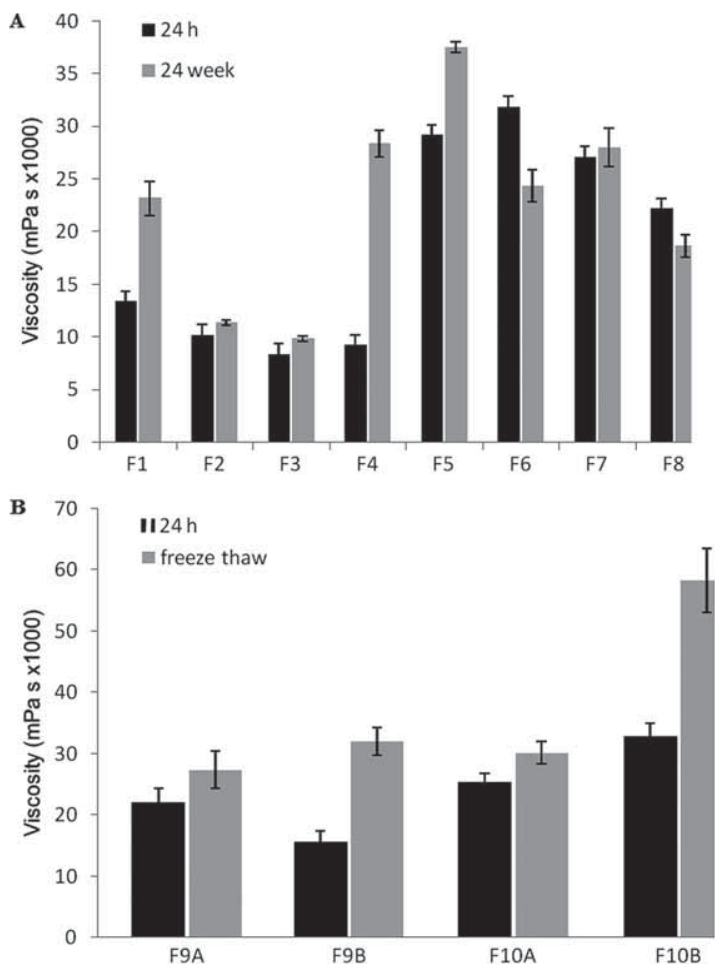

Fig. 1. a) Viscosity of F1-F8 before and after 6 months of storage, b) viscosity of F9A, F9B, F10A and F10B obtained 24 hours after preparation and after the freeze-thaw cycle, respectively (20 rpm). 
in Fig. 1b, after freeze thaw studies the viscosity changes in formulations F9B and F10B, produced by ultrasonication, were higher than in formulations F9A and F10A prepared using magnetic stirring. Greater impact of freeze thaw cycling on F9B and F10B could be dependent on the tendency of the two formulations prepared by the ultrasonication method towards changes in interface stability.

The final acceptance of an emulsion depends on the stability, appearance and functionality of the product (20). In our study, a long-term stability test was carried out at 25 $\pm 1{ }^{\circ} \mathrm{C}$ and $50 \pm 5 \%$ relative humidity on formulations F1-F8, while formulations F9A, F9B, F10A and F10B were subjected to accelerated stability tests. Viscosity measurements and optical microscopic analyses were conducted in order to examine any changes in homogeneity and phase separation. Optical microscopy was used to observe and evaluate the size of droplets individually. Fig. 2 shows the photomicrographs of emulgels F1, F5 and F8 after $24 \mathrm{~h}$ and 6 months. As can be seen from these micrographs, all of the samples showed some degree of droplet coalescence over the entire 6-month period.
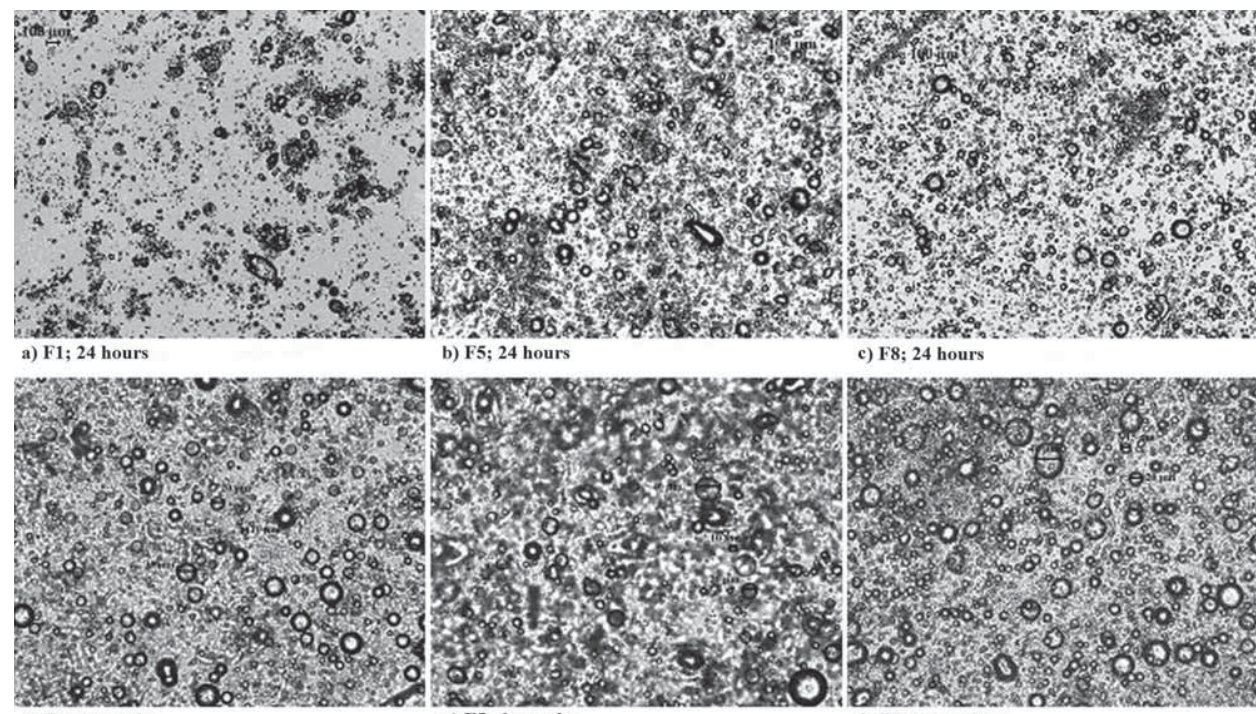
b) $\mathrm{F} 5 ; 24$ hours c) F8; 24 hours

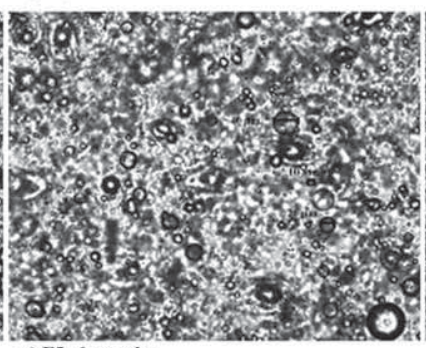

e) F5; 6 months

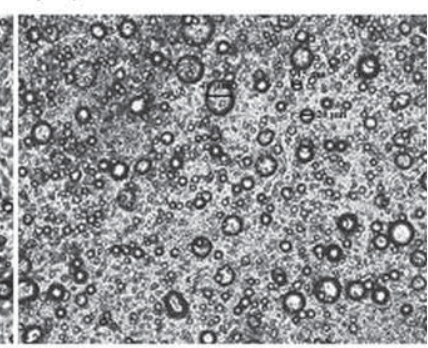

f) F8; 6 months

Fig. 2. Optical microscopy of F1, F5 and F8 ( $\times 10$ magnification) after $24 \mathrm{~h}$ and 6 months, a) F1; 24 h, b) F5; 24 h, c) F8; 24 h, d) F1; 6 months, e) F5; 6 months, f) F8; 6 months.

To evaluate the effect of the increased amount of oil phase on emulgel homogeneity, microscopic analyses were carried out on formulations F9-F10 before and after stability testing (Fig. 3). Ultrasonication process resulted in smaller globules and more homogeneous emulgels were obtained with formulations F9B and F10B. The micrographs before and after accelerated stability tests showed that homogeneity of the formulations did not change and the micrographs can be interpreted as a prediction of long-term stability for emulgel formulations F9A, F9B, F10A and F10B. 
E. Algin Yapar et al.: Design and in vivo evaluation of emulgel formulations including green tea extract and rose oil, Acta Pharm. 63 (2013) 531-543.
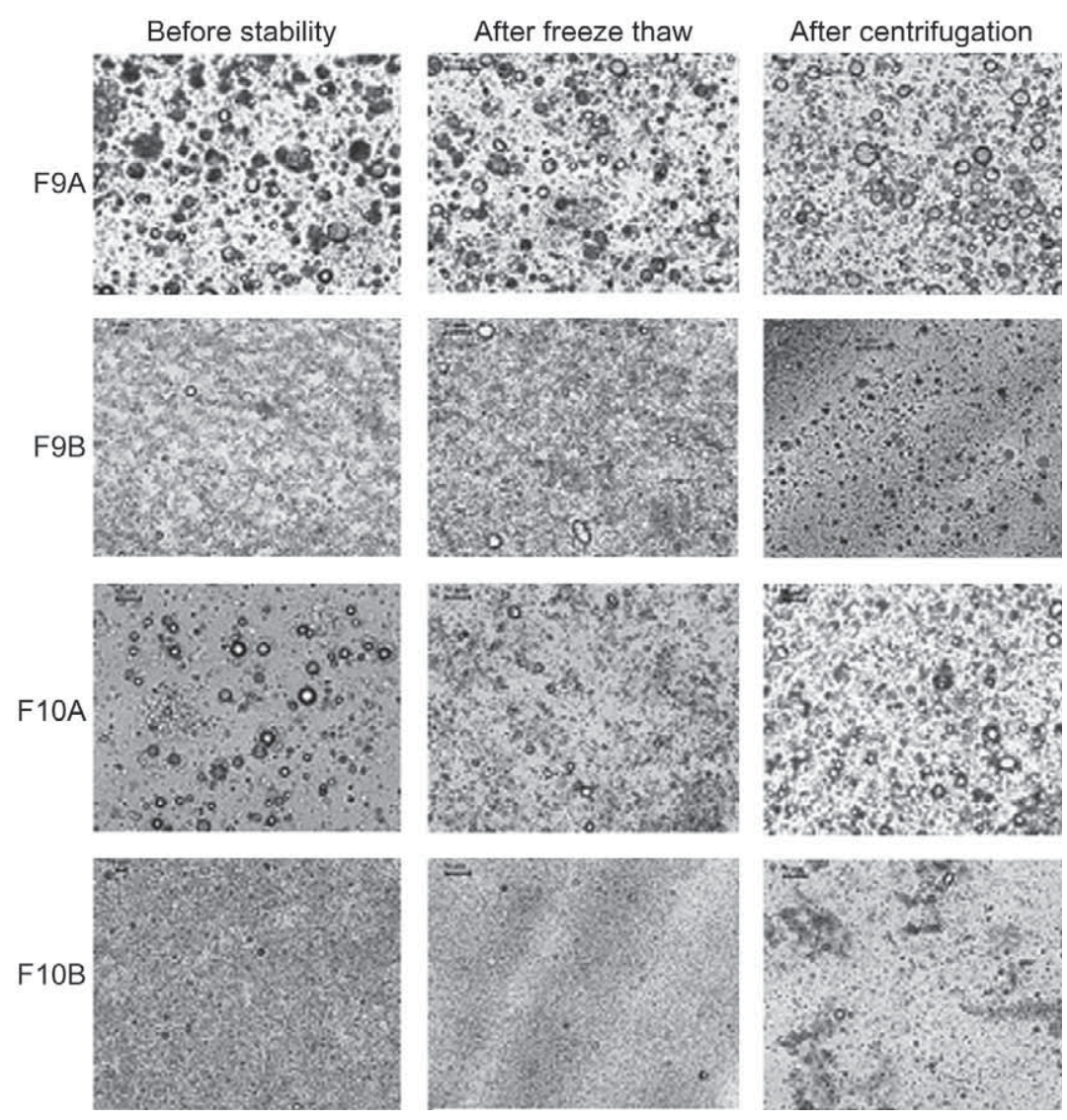

Fig. 3. Optical microscopy of F9A, F9B, F10A and F10B before and after accelerated stability tests (x10 magnification).

It has been reported that a centrifugation process at $1500 \mathrm{~g}(3750 \mathrm{rpm})$ for $5 \mathrm{~h}$ can be used in order to interpret long-term stability of an emulsion (11). Emulgel formulations F9A, F9B, F10A and F10B maintained their consistency and homogeneity after 1 and $5 \mathrm{~h}$ of centrifugation and no phase separation was detected. The emulgel formulation F9A, showing the most promising organoleptic characteristics, spreadability, viscosity and stability, was chosen for in vivo studies. Formulation F5 was used as the Base Cream in in vivo studies.

Noninvasive skin biophysical techniques allowing evaluation of skin care products have found place in cosmetology and dermatology, under actual conditions of use. Hydration and skin barrier function measurements are among the most widely used techniques (22-23). Determination of the hydration state of stratum corneum is generally based on electrical measurements on the skin surface. Corneometer is a well-known and efficient instrument for measuring the hydration of stratum corneum based on the capacitance method (22-24). Water has a crucial role in respect to the normal function of the 
E. Algin Yapar et al.: Design and in vivo evaluation of emulgel formulations including green tea extract and rose oil, Acta Pharm. 63 (2013) 531-543.

epidermal barrier and mechanical properties of skin. Water content of stratum corneum is therefore of particular interest in most cosmetic and dermatologic applications $(23,25)$.

Results of the skin water content measurements after a single application of emulgel formulation F9A and Base Cream averaged over 10 volunteers are represented in Fig. 4a. Maximum value was achieved within $30 \mathrm{~min}$ after application, exhibiting a 1.4 fold increase $(p<0.05)$. Skin water content reached constant values after $180 \mathrm{~min}$ with the emulgel F9A formulation and remained higher than the treatment with Base Cream. The presence of rose oil and green tea extract may have had a positive effect on the increase in skin water content. Green tea extract has been reported to protect against UV irradiation induced photo-aging, photo-immunosuppression, cutaneous erythema, thickening of the epidermis and to prevent oxidative stress and DNA damage (26). Rose oil may interact synergistically with the oily ingredient of the emulgel and help form an effective protective film on the skin surface.

Passive diffusion of water from hydrated layers of the dermis and epidermis toward the superficial stratum corneum layer is defined as TEWL. TEWL measurements can provide a good insight into the efficacy of a new product. Measurement of TEWL is one of the most widely used biophysical methods because it correlates directly with the skin barrier dysfunction and has applications in cosmetology, pharmaceutical, clinical, toxicological, and various other studies $(25,27-29)$. Tewameter is a well-known device capable of measuring TEWL accurately by using the open-chamber method (28).

Results of TEWL measurements after a single application of emulgel F9A and Base Cream averaged over 10 volunteers are given in Fig. 4b. 15 min after the application of
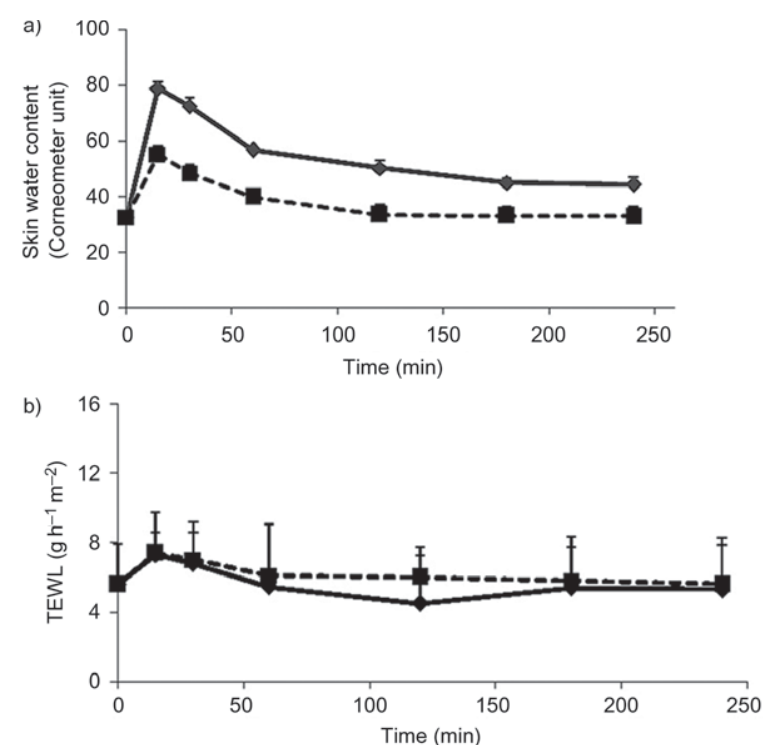

Fig. 4. a) Skin water content measurement after a single application of Emulgel formulation F9A $(\diamond)$ and Base Cream (ם) averaged over 10 volunteers, b) Skin barrier function measurement after a single application of Emulgel formulation F9A and Base cream averaged over 10 volunteers. 
both formulations, TEWL was increased $\left(7.3 \pm 1.2\right.$ and $7.4 \pm 2.4 \mathrm{~g} \mathrm{~h}^{-1} \mathrm{~m}^{-2}$ for F9A and Base Cream, respectively) compared to the baseline $\left(5.6 \pm 2.3 \mathrm{~g} \mathrm{~h}^{-1} \mathrm{~m}^{-2}\right)$ due to evaporation of the water contained in the formulation on contact with the skin surface. The observed TEWL values after single applications of the two formulations were not significantly different in comparison with the baseline.

Long-term application of emulgel F9A resulted in significantly higher skin water content compared to that of untreated skin $(p<0.05)$. The average TEWL value measured after 5 days of emulgel F9A application was considerably lower compared to that of the Base Cream and baseline (Fig. 5). The significant increase in skin humidity proves that stratum corneum hydration depends both on the ability to bind and the ability to retain water (30). As TEWL refers to the total amount of water loss through the skin, lower TEWL values indicate an intact epidermal barrier function that correlates with the normal hydration state of the stratum corneum $(25,27)$.

a)

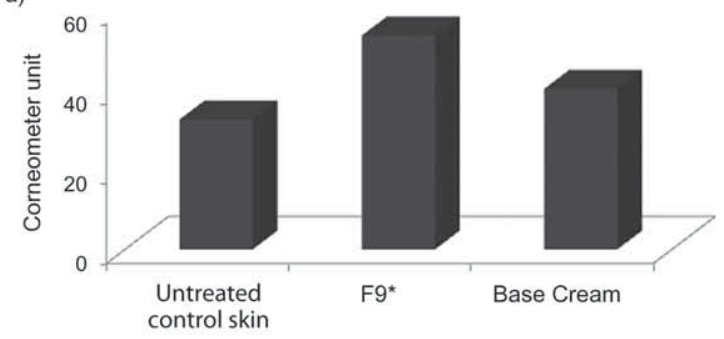

b)

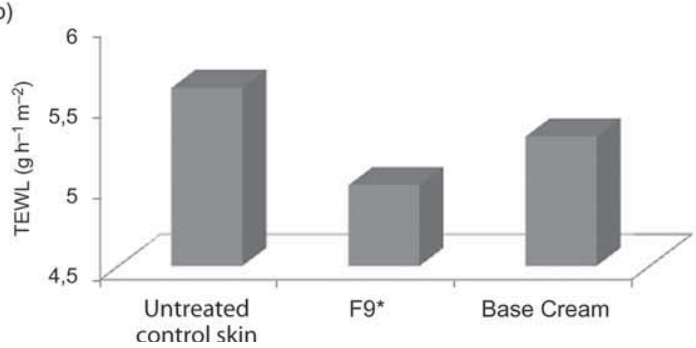

Fig. 5. a) Skin water content, and b) TEWL values averaged over all 5 volunteers after 5 days of application of Emulgel F9A and Base Cream.

\section{CONCLUSIONS}

Based on the results of our study, it can be concluded that the emulgel formulation F9A, including green tea extract and rose oil, was ideal in terms of applicability and stability. It showed a significantly higher hydration effect on human skin by enhancing the skin barrier function. Further investigations should be considered for enhancing the stability of green tea extract and rose oil in the formulation. In vivo studies should be per- 
E. Algin Yapar et al.: Design and in vivo evaluation of emulgel formulations including green tea extract and rose oil, Acta Pharm. 63 (2013) 531-543.

formed under simulated dry or sub erythema UV exposure treatments in order to evaluate the moisturizing and skin barrier protecting effects of this emulgel formulation more accurately.

\section{REFERENCES}

1. A. Sharma, S. Gupta, I. P. Sarethy, S. Dang and R. Gabrani, Green tea extract: possible mechanism and antibacterial activity on skin pathogens, Food Chem. 135 (2012) 672-675; DOI: 10.1016/ j.foodchem.2012.04.143.

2. T. Mahmood and N. Akhtar, Combined topical application of lotus and green tea improves facial skin surface parameters, Rejunevation Res. 16 (2013) 91-97; DOI: 10.1089/rej.2012.1380.

3. S. Hsu, Green tea and skin, J. Am. Acad. Dermatol. 52 (2005) 1049-1059; DOI: 10.1016/j.jaad.2004. 12.044 .

4. C. S. Yang, H. Wang, G. X. Li, Z. Yang, F. Guan and H. Jin, Cancer prevention by tea: evidence from laboratory studies, Pharmacol. Res. 64 (2011) 113-122; DOI: 10.1016/j.phrs.2011.03.001.

5. T. Mahmood, N. Akhtar, B. A. Khan, H. M. Shoaib Khan and T. Saeed, Changes in skin mechanical properties after long term application of cream containing green tea extract, Aging Clin. Exp. Res. 23 (2011) 333-336.

6. J. I. Silverberg, J. Jagdeo, M. Patel, D. Siegel and N. Brody, Green tea extract protects human skin fibroblasts from reactive oxygen species induced necrosis, J. Drugs Dermatol. 10 (2011) 1096-1101.

7. A. Rasheed, S. N. Shama, J. M. Joy, B. S. Reddy and C. Roja, Formulation and evaluation of herbal anti-acne moisturizer, Pak. J. Pharm. Sci. 25 (2012) 867-870.

8. F. Casetti, U. Wölfle, W. Gehring and C. M. Schempp, Dermocosmetics for dry skin: a new role for botanical extracts, Skin Pharmacol. Physiol. 24 (2011) 289-293; DOI: 10.1159/000329214.

9. S. Ulusoy, G. Bosgelmez-Tinaz and H. Seçilmis-Canbay, Tocopherol, carotene, phenolic contents and antibacterial properties of rose essential oil, hydrosol and absolute, Curr. Microbiol. 59 (2009) 554-558; DOI: 10.1007/s00284-009-9475-y.

10. A. Wei and T. Shibamoto, Antioxidant activities of essential oil mixtures toward skin lipid squalene oxidized by UV irradiation, Cutan. Ocul. Toxicol. 26 (2007) 227-233; DOI: 10.1080/15569520 701224501.

11. T. Hongratanaworakit, Relaxing effect of rose oil on humans, Nat. Prod. Commun. 4 (2009) 291296.

12. A. R. Silva, C. Seidl, A. S. Furusho, M. M. Boeno, G. C. Dieamant and A. M. Weffort-Santos, In vitro evaluation of the efficacy of commercial green tea extracts in UV protection, Int. J. Cosmet. Sci. 35 (2013) 69-77; DOI: 10.1111/ics.12006.

13. P. Kullavanijaya and H. W. Lim, Photoprotection, J. Am. Acad. Dermatol. 52 (2005) 937-958; DOI: 10.1016/j.jaad.2004.07.063.

14. M. P. Lupo, Antioxidants and vitamins in cosmetics, Clin. Dermatol. 19 (2001) 467-473.

15. S. E. Dal Belo, L. R. Gaspar, P. M. Maia Campos and J. P. Marty, Skin penetration of epigallocatechin-3-gallate and quercetin from green tea and Ginkgo biloba extracts vehiculated in cosmetic formulations, Skin Pharmacol. Physiol. 22 (2009) 299-304; DOI: 10.1159/000241299.

16. P. V. Bhanu, V. Shanmugam and P. K. Lakshmi, Development and optimization of novel diclofenac emulgel for topical drug delivery, Pharmacie Globale 9 (2011) 1-4.

17. A. Sakulpanich and W. Gritsanapan, Extraction method for high content of anthraquinones from cassia fistula pods, J. Health Res. 22 (2008) 167-172. 
E. Algin Yapar et al.: Design and in vivo evaluation of emulgel formulations including green tea extract and rose oil, Acta Pharm. 63 (2013) 531-543.

18. S. Ghosh and D. Rousseau, Freeze-thaw stability of water-in-oil emulsions, J. Colloid. Interface Sci. 339 (2009) 91-102; DOI: 10.1016/j.jcis.2009.07.047.

19. D. Vasiljevic, J. Parojcic, M. Primorac and G. Vuleta, An investigation into the characteristics and drug release properties of multiple $\mathrm{W} / \mathrm{O} / \mathrm{W}$ emulsion systems containing low concentration of lipophilic polymeric emulsifier, Int. J. Pharm. 309 (2006) 171-177; DOI: 10.1016/j.ijpharm. 2005.11.034.

20. M. M. Rieger, Emulsions, in Theory and Practice of Industrial Pharmacy (Eds. L. Lachman, H. A. Lieberman and J. L. Kanig), Verlag Lea and Febiger Publishing, Philadelphia 1986, pp. 527-530.

21. J. Pinnagoda, R. A. Tupker, T. Agner and J. Serup, Guidelines for transepidermal water loss (TEWL) measurement, Contact Dermatitis 22 (1990) 164-178; DOI: 10.1111/j.1600-0536.1990.tb 01553.x

22. Y. Cheng, Y. Dong, C. Wang, N. Su, Y. Sun, J. Liu, H. Zheng, A. Schrader, M. Rohr and W. Liu, Protection effect of cosmetics on human skin under simulated rigorous environment, Skin. Res. Technol. 14 (2008) 45-52; DOI: 10.1111/j.1600-0846.2007.00260.x.

23. G. Betz, A. Aeppli, N. Menshutina and H. Leuenberger, In vivo comparison of various liposome formulations for cosmetic application, Int. J. Pharm. 296 (2005) 44-54; DOI: 10.1016/j.ijpharm. 2005.02.032.

24. P. Clarys, R. Clijsen, J. Taeymans and A. O. Barel, Hydration measurements of the stratum corneum: comparison between the capacitance method (digital version of the Corneometer CM $825^{\circledR}$ ) and the impedance method (Skicon-200EX ${ }^{\circledR}$ ), Skin. Res. Technol. 18 (2012) 316-323; DOI: 10.1111/j.1600-0846.2011.00573.x.

25. J. Du Plessis, A. Stefaniak, F. Eloff, S. John, T. Agner, T. C. Chou, R. Nixon, M. Steiner, A. Franken, I. Kudla and L. Holness, International guidelines for the in vivo assessment of skin properties in non-clinical settings: part 2. Transepidermal water loss and skin hydration, Skin Res. Technol. 1 (2013) 1-10; DOI: 10.1111/srt.12037.

26. P. K. Mukherjee, N. Maity, N. K. Nema and B. K. Sarkar, Bioactive compounds from natural resources against skin aging, Phytomedicine 19 (2011) 64-73; DOI: 10.1016/j.phymed.2011.10.003.

27. R. Darlenski, S. Sassning, N. Tsankov and J. W. Fluhr, Noninvasive in vivo methods for investigation of the skin barrier physical properties, Eur. J. Pharm. Biopharm. 72 (2009) 295-303; DOI: 10.1016/j.ejpb.2008.11.013.

28. B. Sotoodian and H. I. Maibach, Noninvasive test methods for epidermal barrier function, Clin. Dermatol. 30 (2012) 301-310; DOI: 10.1016/j.clindermatol.2011.08.016.

29. K. A. Walters and M. S. Roberts, Skin Structure, Pharmaceuticals, Cosmetics and the Efficacy of Topically Applied Agents, in Dermatologic, Cosmeceutic and Cosmetic Development, Informa Healthcare, New York 2008, pp. 1-10.

30. M. Breternitz, D. Kowatzki, M. Langenauer and P. Elsner, Placebo controlled, double blind, randomized, prospective study of a glycerol based emollient on eczematous skin in atopic dermatitis: biophysical and clinical evaluation, Skin Phamacol. Physiol. 21 (2008) 39-45; DOI: 10.1159/ 000111134. 